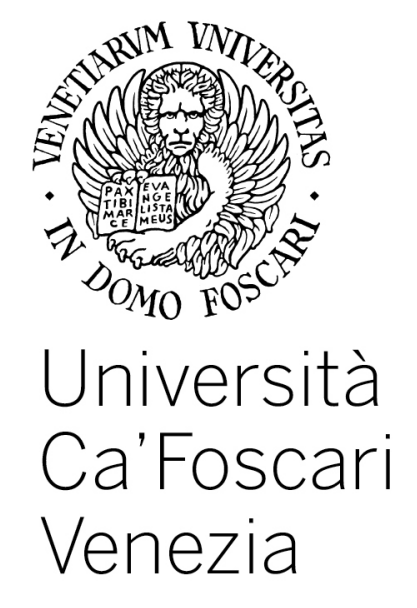

Department
of Management

Working Paper Series

Francesco Mason and Andrea Borghesan

Circuits of Iterated Foata Maps

Working Paper n. 25/2014 October 2014

ISSN: 2239-2734

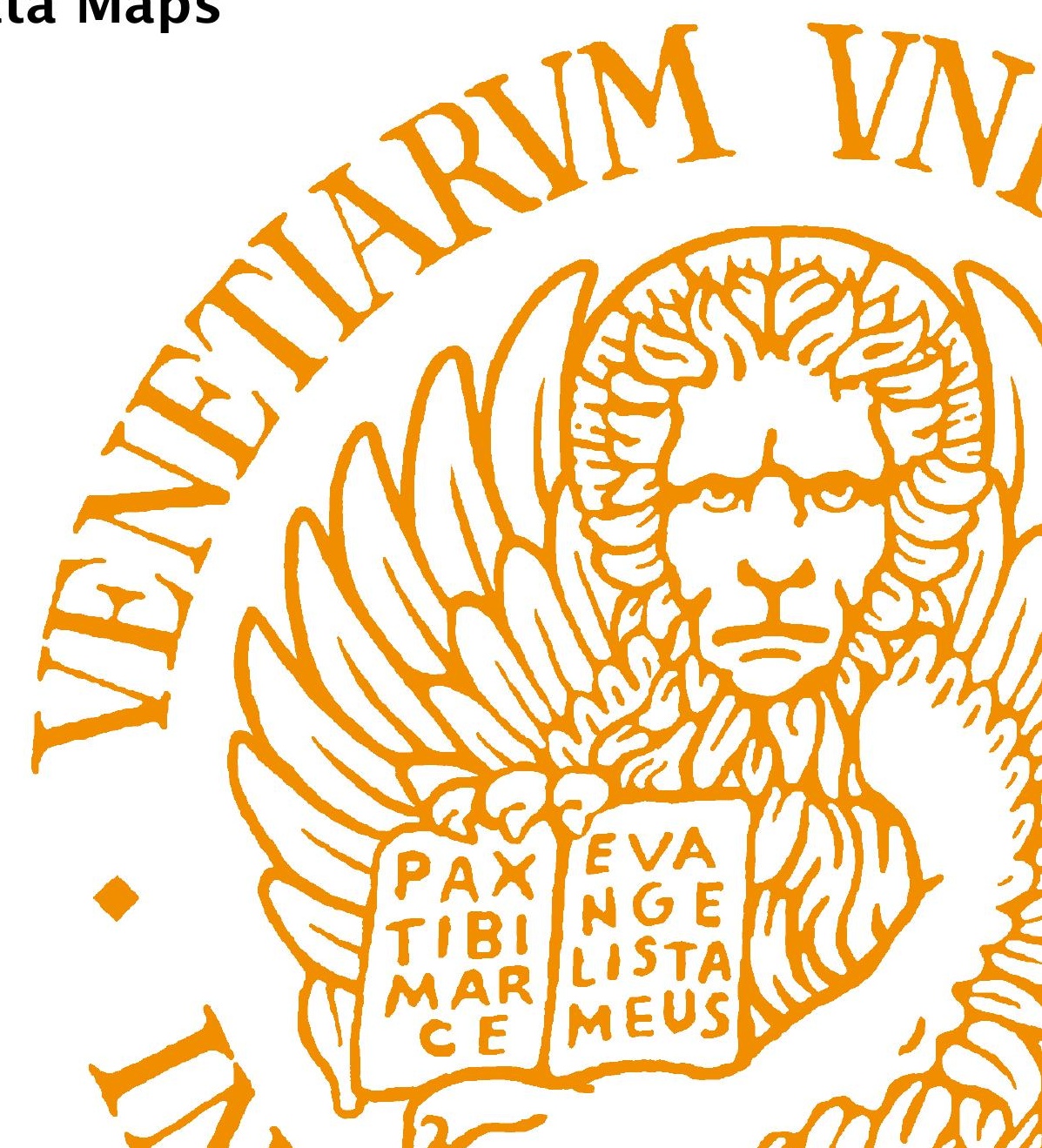


This Working Paper is published under the auspices of the Department of Management at Università $\mathrm{Ca}^{\prime}$ Foscari Venezia. Opinions expressed herein are those of the authors and not those of the Department or the University. The Working Paper series is designed to divulge preliminary or incomplete work, circulated to favour discussion and comments. Citation of this paper should consider its provisional nature. 


\title{
Circuits of Iterated Foata Maps
}

\author{
Francesco Mason \\ $<$ fmason@unive.it $>$ \\ Dept. of Management \\ University of Venice
}

\author{
ANDREA BorghesAN \\ $<$ borg@unive.it> \\ Dept. of Management \\ University of Venice
}

(April 2014)

\begin{abstract}
In this paper we will consider Foata map as a function which associates another permutation, known as $F(\sigma)$, to each permutation $\sigma=\sigma_{1} \sigma_{2} \cdots \sigma_{m}$ of the integers $\{1,2, \ldots, m\}$, in such a way that the number of cycles of $\sigma$ is equal to the number of left to right minima of $F(\sigma)$. We will analyze the behaviour of the iterated maps, $F^{2}(\sigma)=F(F(\sigma)), F^{3}(\sigma), \ldots$ and so on. It is clear that given a permutation $\sigma$, positive integer numbers $k$ exist such that $F^{k}(\sigma)=\sigma$. Consider the smallest of these numbers and call it $n$. We shall call the (ordered) set of permutations $C(\sigma)=\left\{\sigma, F(\sigma), \ldots, F^{n-1}(\sigma)\right\}$ a Foata circuit and $n$ its length. The length of Foata circuits is the main concern of this paper: to our knowledge, no study has been devoted to this subject yet.

Experimental results are quite surprising. In computable cases, generally speaking, there are one (at most two) circuit(s) of very large length (up to $85 \%$ of the total number of permutations) and a number of little circuits, even of some units of components. In this paper we will give a list of these circuits for permutations up to 11 elements. However these data give rise to some conjectures, difficult to verify: the investigation on permutations of more than 10 elements is a challenge and the case of $m=11$ is a demonstration of this issue! At the same time, in this paper we will also discuss some properties of 'short' circuits, i.e. the ones consisting in 2,3 or 4 permutations.
\end{abstract}

Keywords: Circuits, Foata maps, Permutations.

JEL Classification Numbers: C43, C63.

MathSci Classification Numbers: 05E18.

\section{Correspondence to:}

$\begin{array}{ll}\text { Francesco Mason } & \begin{array}{l}\text { Dept. of Management, University of Venice } \\ \text { S.Giobbe, Cannaregio 873 }\end{array} \\ & 30121 \text { Venice, ITALY } \\ \text { Phone: } & {[++39](041)-234-6916} \\ \text { Fax: } & {[++39](041)-234-7444} \\ \text { E-mail: } & \text { fmason@unive.it }\end{array}$




\title{
CIRCUITS OF ITERATED FOATA MAPS
}

\author{
Andrea Borghesan - Francesco Mason \\ Università degli Studi Ca' Foscari di Venezia
}

\section{Introduction: basic definitions and hypothesis.}

Let be $\sigma=\sigma_{1} \sigma_{2} \ldots \sigma_{\mathrm{m}}$ a permutation whose elements are the ones in the set $\{1$, $2, \ldots, m\}$. The Foata map we will consider in this paper is defined as follows:

1) Find the cycles in $\sigma$.

2) Write every cycle in such a way that the first element is the minimum of the elements of the cycle. Then write all the cycles in $\sigma$ in order of decreasing minima.

3) Erase the symbols of separation between cycles, in order to get a new permutation. This will be called the Foata map (by decreasing minima) of the given permutation.

Foata map was originally introduced in order to recognize the cycles in $\sigma$ through left to right minima of $F(\sigma)$.

A left to right minimum is each element $\sigma_{i}$ for which $\sigma_{j}>\sigma_{i}$ for all $j<i$. By definition, $\sigma_{1}$ is always a left to right minimum.

The main property of a Foata transformation is the following one (see for example [1], [2]):

The number of cycles of a permutation $\sigma$ is equal to the number of local left to right minima in the Foata map $F(\sigma)$.

The Foata map is a bijection: the inverse transformation, from $\sigma$ to $\rho=F^{-1}(\sigma)$ is quite easy to perform (see [2], Transition Lemma).

The Foata map may be defined in a different way assuming that every cycle is written beginning with the greatest element. In this case we will dispose cycles in increasing order of maxima. The theorem above can be reformulated in an evident way.

However in what follows, however, we will always consider the Foata map as the one satisfying the 'minima decreasing' criterium.

As long as

a) the Foata map exists for every permutation;

b) the permutations on a given number $m$ of elements is a finite number (i.e. $\mathrm{m} !)$; 
c) Foata map is a bijection,

the following statements hold:

- given a permutation $\sigma$, let $n$ be the smallest positive integer $(\leq \mathrm{m} !)$ for which $\mathrm{F}^{\mathrm{n}}(\sigma)=\sigma$ : in this way, $\sigma, F(\sigma), \mathrm{F}^{2}(\sigma), \ldots, \mathrm{F}^{\mathrm{n}-1}(\sigma)$ are all different and the Foata transform produces a circuit of permutations; we shall call every circuit of this kind a Foata circuit; $\mathrm{n}$ will be called the length of the circuit;

- every permutation belongs to one and only one Foata circuit.

In this way, for every fixed $\mathrm{m}$, the $\mathrm{m}$ ! permutations of the elements $1,2, \ldots, \mathrm{m}$, are partitioned in a certain number of Foata circuits.

Every Foata circuit can be represented (generated) by one of its permutations. Whenever possible, we shall identify a circuit by the lexicographically lest permutation.

\section{Empirical results about Foata circuits.}

It is easy to see that:

- if $m=1$, (trivial case), we have only one Foata circuit of length1;

- if $\mathrm{m}=2$, we have a Foata circuit of the two permutations 12 and 21 ;

- if $m=3$, the six permutations belong to a unique Foata circuit.

In the cases studied, for $m \geq 4$, the number of Foata circuits is greater than 1 (and always an even number!).

For $m=4$ there are two Foata circuits, containing respectively 15 and 9 elements. The one containing 15 elements has, as its (lexicographically) smallest generator the permutation 1234; the other one has as smallest generator 1243.

The case $m=5$ gives a quite surprising result. This particular result arose our curiosity and motivated our subsequent investigations. In fact, the 120 permutations are partitioned in two circuits, the first one of 117 elements and the second one consisting of the remaining 3 elements.

The following tables report the list of the Foata circuits when $5 \leq \mathrm{m} \leq 11$. For every $\mathrm{m}$, we give the length of each circuit and the corresponding smallest generator.

$m=5(m !=120)$

\begin{tabular}{|c|c|}
\hline 117 & 12345 \\
\hline 3 & 21354 \\
\hline
\end{tabular}

$m=6(m !=720)$

\begin{tabular}{|c|c|}
\hline 694 & 123456 \\
\hline 17 & 145326 \\
\hline 5 & 236415 \\
\hline 4 & 164523 \\
\hline
\end{tabular}


$m=7(m !=5040)$

\begin{tabular}{|r|r|}
\hline 4804 & 1234567 \\
\hline 197 & 1234675 \\
\hline 21 & 1254673 \\
\hline 8 & 1253647 \\
\hline 7 & 2451673 \\
\hline 3 & 2637145 \\
\hline
\end{tabular}

$m=8 \quad(m !=40320)$

\begin{tabular}{|r|r|}
\hline 34864 & 12345678 \\
\hline 2426 & 12357684 \\
\hline 2033 & 12358764 \\
\hline 574 & 12768453 \\
\hline 213 & 12485736 \\
\hline 196 & 12845376 \\
\hline 7 & 13874265 \\
\hline 7 & 14825763 \\
\hline
\end{tabular}

$m=9 \quad(m !=362880)$

\begin{tabular}{|c|l|}
\hline 166095 & 123459678 \\
\hline 139800 & 123456789 \\
\hline 23281 & 123456798 \\
\hline 13337 & 123468759 \\
\hline 10768 & 123456987 \\
\hline 8568 & 123467589 \\
\hline 531 & 123574689 \\
\hline 362 & 125698347 \\
\hline 106 & 124736895 \\
\hline 23 & 134658297 \\
\hline 5 & 183742695 \\
\hline 4 & 196754238 \\
\hline
\end{tabular}

$m=10 \quad(m !=3628800)$

\begin{tabular}{|r|r|}
\hline 3223086 & 12345678109 \\
\hline 208809 & 12345678910 \\
\hline 80379 & 12345671098 \\
\hline 75045 & 12345698710 \\
\hline 23513 & 12347108956 \\
\hline 11331 & 12346971085 \\
\hline 2856 & 12376954108 \\
\hline 1809 & 12364875910 \\
\hline 1207 & 12364957108 \\
\hline 416 & 12491065738 \\
\hline
\end{tabular}




\begin{tabular}{|c|c|}
\hline 292 & 12631058497 \\
\hline 57 & 12569374108 \\
\hline
\end{tabular}

$m=11 \quad(m !=39916800)$

\begin{tabular}{|r|l|}
\hline 28022027 & 1234567810911 \\
\hline 5073003 & 1234567981110 \\
\hline 2247005 & 1234567891110 \\
\hline 1455309 & 1234567891011 \\
\hline 1141445 & 1234567911108 \\
\hline 845854 & 1234568107911 \\
\hline 822240 & 1234567111089 \\
\hline 110743 & 1234651079118 \\
\hline 94762 & 1234659711810 \\
\hline 46888 & 1234589106711 \\
\hline 19516 & 1234611579108 \\
\hline 15437 & 1234681091157 \\
\hline 10890 & 1234711105896 \\
\hline 10350 & 1235104711968 \\
\hline 777 & 1235106411987 \\
\hline 224 & 1238107119456 \\
\hline 169 & 1298457116310 \\
\hline 161 & 1231058741169 \\
\hline & \\
\hline
\end{tabular}

In the case $m=12$ (in which there are 479001600 permutations), we began testing two generators, i.e. the identity permutation

123456789101112

and the permutation obtained exchanging the last two elements in the identity permutation:

123456789101211.

This was suggested us by some of the preceding cases, in which the identity permutations and the other one, obtained exchanging the last two elements, give rise to two different circuits.

Indeed, this is also the case for $m=12$ : the permutations above produced the first two cycles (see the following table), which contain together 474149644 permutations. This fact allowed, in searching other circuits, to reduce successive computing time, by stopping every search when the circuit under investigation demonstrated to be longer than 4900000 permutations.

We proceeded the search changing the generator systematically on the basis of a lexicographical ordering of permutations on 12 elements.

In this way, we found very quickly a circuit of near 450000 elements and then, with 85 trials, a circuit of 746402 permutations and with less than 200 trials, a circuit of more than 3 millions permutations. The $336^{\text {th }}$ instance gave the circuit with 109369 permutations, while the $771^{\text {th }}$ gave the one with 21294 permutations. The results at the moment are as follows: 


\begin{tabular}{|c|c|}
\hline 261048435 & 123456789101211 \\
\hline 213101209 & 123456789101112 \\
\hline 3135130 & 123456810111297 \\
\hline 746402 & 123456711108912 \\
\hline 454788 & 123456789111012 \\
\hline$\ldots$ & $\ldots$ \\
\hline 109369 & 123456911121087 \\
\hline$\ldots$ & 123457610811912 \\
\hline$\ldots$ & $\ldots$ \\
\hline
\end{tabular}

We cannot be sure that there are not any other circuits longer than 100000 units, so the position of the 6-th and 7-th circuits of the list is not yet defined (other circuits longer than 100000 elements can exist).

However, the remaining permutations are 385003 , about $0.1 \%$ of the total.

In our analysis, which tests values up to 12 , we have an increasing trend in the number of circuits (but pay attention to the two cases, $m=9$ and $m=10$, both with 12 circuits). The identity permutation

$$
123 \ldots(m-1) m
$$

belongs to the longest circuit for $m \leq 8$, while for $m \geq 9$ this is not true. Moreover, as we stated above, for $m \geq 9$ if we exchange the last two elements in the identity permutation, we will get a generator of a different circuit.

The experimental evidence suggests some conjectures:

- an increasing (or not decreasing) number of Foata circuits as m increases;

- one or two - at most, see cases $m=9$ and $m=12$ - very large circuits, containing more than $85 \%$ of all the permutations;

- $\quad$ an increasing trend in the number of elements in the shortest circuit, when $m \geq 9$

Some other properties will be investigated in the following section.

\section{Theoretical results about 'short' circuits.}

The proof of the following statements is given in the Appendix.

Lemma 3.1. For every $m>1$ and $\sigma, F(\sigma) \neq \sigma$.

As a consequence, when $m>1$, every Foata circuit contains two permutations or more.

Lemma 3.2 If $\sigma=1 \sigma_{2} \sigma_{3} \ldots \sigma_{m}$, then $F(\sigma)=\rho_{1} \rho_{2} \ldots \rho_{m-1} 1$.

Conversely, if $\sigma=\sigma_{1} \sigma_{2} \ldots \sigma_{\mathrm{m}-1} 1$, then $\rho=\mathrm{F}^{-1}(\sigma)=1 \rho_{2} \ldots \rho_{\mathrm{m}}$.

Lemma 3.3 If $\sigma=\sigma_{1} \sigma_{2} \ldots \sigma_{m-1} m$, then $F(\sigma)=m \rho_{2} \ldots \rho_{m-1} \rho_{m}$.

Conversely, if $\sigma=m \rho_{2} \ldots \rho_{m-1} \rho_{m}$, then $\rho=F^{-1}(\sigma)=\rho_{1} \rho_{2} \ldots m$.

Lemma 3.4 If $\sigma=1 \sigma_{2} \ldots \sigma_{m-1} m$, then $F(\sigma)=m \rho_{2} \ldots \rho_{m-1} 1$. (And conversely). 
Corollary 3.1 If $\sigma=1 \sigma_{2} \ldots \sigma_{\mathrm{m}-1} \mathrm{~m}$, then $\rho=\mathrm{F}^{-1}(\sigma)=\rho_{1} \rho_{2} \ldots \rho_{\mathrm{m}-1} 1$.

Lemma 3.5 If $\sigma=m \sigma_{2} \ldots \sigma_{m-1} 1$, then $\rho=F(\sigma)=a \rho_{2} \rho_{3} \ldots 1 \mathrm{~m}$ (obviously, $a \neq 1$, $\mathrm{a} \neq \mathrm{m}$ ); moreover $\tau=\mathrm{F}^{2}(\sigma)=\mathrm{m} \tau_{2} \tau_{3} \ldots 1 \mathrm{a} \ldots \mathrm{m}-1$, where it possibly can results $a=m-1$.

Corollary 3.2 In a Foata circuit, if

then

$$
\sigma=1 \sigma_{2} \sigma_{3} \ldots \mathrm{m}
$$

$$
\begin{aligned}
& F(\sigma)=m \rho_{2} \ldots \rho_{m-1} 1 \\
& \mathrm{~F}^{2}(\sigma)=\mathrm{a} \tau_{2} \ldots \tau_{\mathrm{m}-2} 1 \mathrm{~m}
\end{aligned}
$$

$a \neq 1, m ;$ moreover

whereas $F^{3}(\sigma)$ has a structure

$$
\begin{aligned}
& \mathrm{F}^{-2}(\sigma)=1 v_{2} \ldots v_{\mathrm{m}} ; \\
& \mathrm{F}^{-1}(\sigma)=\mu_{1} \mu_{2} \ldots \mu_{\mathrm{m}-1} 1 ;
\end{aligned}
$$

in which it can be $\mathrm{a}=\mathrm{m}-1$.

$$
F^{3}(\sigma)=m \ldots 1 \text { a } \ldots m-1
$$

Corollary 3.3 In a Foata circuit, every couple of consecutive permutations

Is followed by a couple of permutations

$$
F^{h}=1 \mu_{2} \ldots \mu_{m}, F^{h+1}=v_{1} \ldots v_{m-1} 1
$$

$$
F^{h+2}=\rho_{1} \ldots \rho_{m-1} m, F^{h+3}=m \tau_{2} \ldots \tau_{m}
$$

Lemma 3.6 Whenever $m>2$, a Foata circuit of length $n=3$ cannot contain permutations in which the first element is 1 .

The only known case of a 2-length circuit is $m=2$. We conjecture that this will be the unique case. We did not succeeded in proving this result. However, it is possible to prove

Lemma 3.7 Circuits of length 2, if any, cannot contain permutations beginning with $1,2, m-1, m$ nor permutations in which the last element is $1,2, m$.

\section{The challenge of the hidden permutations: discovering short circuits.}

In order to detect all the Foata circuits for a given $\mathrm{m}$, we followed three different techniques. The main problem was to manage the total number of permutations and, particularly, given a set of circuits, to discover permutations not yet belonging to any of these circuits. The task was relatively easy for large circuits. But it became more difficult when we tried to identify the shortest circuits. We could easily establish that they exist, but, when $m=10$ or more, it was very problematic, to identify with precision their length and to identify a generator.

The simplest idea, we performed for $m \leq 9$, consisted in the building of the list of all the permutations and then in progressively deleting the permutations of found circuits. The remaining permutations, obviously, must belong to circuits not yet found. Assuming one of these as a new generator, we got a new circuit and so, step by step, all the permutations were classified. 
The procedure can be represented by the following pseudo-algorithm:

1. Build all the permutations and save them in a database "permutations".

2. Repeat

2.1 Read the first permutation $\sigma$ in the data base.

2.2 Use $\sigma$ in order to build a circuit.

2.3 Delete all the permutations belonging to the circuit from the $\mathrm{db}$ "permutations".

3. Until permutations in the database "permutations" exist.

This release suffers from some limits. Generally speaking, it is not necessary to build all the permutations in order to find all the circuits, so in the above algorithm the needed time is not optimized. In particular, the deletion of already met permutations is very time consuming.

More efficient, and tested in case $m=10$, is the following release:

1. Build recursively a permutation

2.1 if the permutation does not belong to any of the circuits so far detected

2.1.1 build the corresponding circuit

2.1.2 save this circuit

2.2 else, go to 1 .

2.3 finish when the global number of permutations in the detected circuits is $\mathrm{m}$ !

This second release may appear heavy and time wasting, when we search if a permutation belongs to an already detected circuit. However, once we have detected some of the longest circuits, and known that q permutations have not yet been classified, we can test if a new permutation can generate a new circuit by stopping the search after $q+1$ iterations of the Foata map.

In order to reduce the search time, we build a third algorithm. The main difference between this new algorithm and the preceding one is that we consider only permutations in which the first element is 1 . This is motivated by the empirical evidence that the shortest circuits, when $m=10$ contained more than 50 permutations: if this represents a general trend, for $m>10$, every circuit contains permutations beginning with the element 1 .

Indeed, this third procedure when applied to $m=11$ revealed all the Foata circuits. The question is open about the detection of all circuits when $m=12$ or more.

\section{Some observations and conclusions}

The very high difference between the numbers of permutations in various circuits, makes somewhat problematic to identify some property that characterizes the permutations belonging to the same circuit.

If the main interest in Foata map is to give a correspondence between cycles and left to right minima (or left to right maxima) the two Foata maps (obtained by 'decreasing minima' or 'increasing maxima') may be considered equivalent. However the properties of circuits raising from the two kinds of maps are very different. Circuits generated from 'increasing maxima criterion' are generally very short. 
On the contrary, long circuits, such the ones obtained in the 'decreasing minima' method, may be interesting in other contests. We think to a possible use in a simulation framework, with some care in order to avoid the correlations between adjacent permutations in the same circuit due to the properties underlying some of the Lemmas and Corollaries in section 3. However, we leave this issue to further deeper researches.

\section{Appendix}

Proof of Lemma 3.1. Consider $\sigma=\sigma_{1} \sigma_{2} \ldots \sigma_{m}$ and $F(\sigma)=\rho_{1} \rho_{2} \ldots \rho_{m}$. If $\sigma_{1}=1$, then $\rho_{m}=1$ (and consequently $\rho_{1} \neq 1$ ). On the contrary, if $\sigma_{1} \neq 1$, then in $\sigma$ we have a cycle of the kind $\left(1 \sigma_{1} \ldots\right)$ which will be the last cycle in $F(\sigma)$ so in $F(\sigma)$ the element $\sigma_{1}$ cannot be in the first position.

Proof of Lemma 3.2. It is quite evident: the element 1 in $\sigma$ will be a cycle by itself and so it will be transposed in the last position in $F(\sigma)$ and vice versa.

Proof of Lemma 3.3 The element $m$ in $\sigma$ is a cycle (a loop) and in $F(\sigma)$ it will be in the first position (because it achieves the maximum of the minima). Vice versa, the position of the element $m$ in $F(\sigma)$ can be originated only from the position of $m$ in the first place of $\sigma$.

Proof of Lemma 3.4. It is an immediate consequence of the preceeding two Lemmas.

Proof of Corollary 3.1. It is a particular case of Lemma 3.2.

Proof of Lemma 3.5. The permutation $\sigma$ contains the cycle $(1 \mathrm{~m})$, which will be the last one in $F(\sigma)$. Denoting with ' $a$ ' the first element in $F(\sigma)$, in $F^{2}(\sigma)$ we will have: $\mathrm{m}$, as first element (it is a loop in $\mathrm{F}(\sigma)$ ); the couple $(1 \mathrm{a})$ somewhere in $\mathrm{F}^{2}(\sigma)$.

Proof of Corollary 3.2. and 3.3 They are consequences of the above Lemmas.

Proof of Lemma 3.6. Effectively, from an empirical point of view, the property holds for $m \leq 4$, so we can suppose $m \geq 5$. From the preceding Lemmas and Corollaries, in a Foata circuit, a permutation $\sigma=1 \sigma_{2} \sigma_{3} \ldots \sigma_{\mathrm{m}}$ is followed by other three permutations in such a way that we get the scheme:

$$
\begin{gathered}
\sigma=1 \ldots \mathrm{a} \\
\mathrm{F}(\sigma)=\mathrm{b} \ldots \mathrm{1} \\
\mathrm{F}^{2}(\sigma)=\mathrm{c} \ldots \mathrm{m} \\
\mathrm{F}^{3}(\sigma)=\mathrm{m} \ldots \mathrm{d} .
\end{gathered}
$$

We prove that these four permutations are always different.

Obviously, $a \neq 1, b \neq 1 ; \sigma \neq F(\sigma), \quad F(\sigma) \neq F^{2}(\sigma), \quad \sigma \neq F^{3}(\sigma), F^{2}(\sigma) \neq F^{3}(\sigma)$. Consider now two cases: $a \neq m$ and $a=m$.

i) If $a \neq m, \sigma \neq F^{2}(\sigma)$, but we also have $F(\sigma) \neq F^{3}(\sigma)$, because it is impossible $b$ $=\mathrm{m}$ (this would induce $\mathrm{a}=\mathrm{m}$ );

ii) If $\mathrm{a}=\mathrm{m}$, the above scheme becomes:

$$
\begin{gathered}
\sigma=1 \ldots \mathrm{m} \\
F(\sigma)=\mathrm{m} \ldots 1 \\
\mathrm{~F}^{2}(\sigma)=\mathrm{c} \ldots 1 \mathrm{~m} \\
\mathrm{~F}^{3}(\sigma)=\mathrm{m} \ldots \mathrm{d},
\end{gathered}
$$


so $\sigma \neq F^{2}(\sigma)$. On the other side, $F^{2}(\sigma)$ contains a cycle of the kind (1 c ... m-1)

and consequently the element 1 cannot be in the last position in $\mathrm{F}^{3}(\sigma)$. In this way we can assert $d \neq 1$ and $F^{3}(\sigma) \neq F(\sigma)$.

\section{Bibliography}

[1] J.L Baril - Gray code fo permutations with a fixed number of left to right minima - Ars Combinatoria Vol CXI pp 225-239, 2013.

[2] M.Bòna - Combinatorics of permutations - Chapman \& Hall 2004. 